\title{
Supporting information for: \\ Application of the tube model to explain the unexpected decrease in polymer bending energy induced by knot formation
}

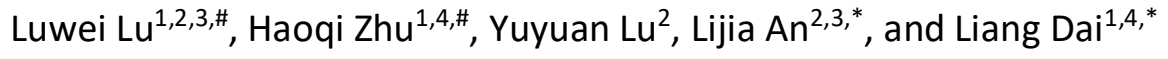 \\ ${ }^{1}$ Department of Physics, City University of Hong Kong, Hong Kong, P.R. China \\ ${ }^{2}$ State Key Laboratory of Polymer Physics and Chemistry, Changchun Institute of Applied \\ Chemistry, Chinese Academy of Sciences, Changchun 130022, P.R. China \\ ${ }^{3}$ University of Science and Technology of China, Chinese Academy of Sciences, Hefei, P.R. China \\ ${ }^{4}$ Shenzhen Research Institute, City University of Hong Kong, Shenzhen, China
}

S1. Additional simulation results and analysis of long-tube confinement

S2. Additional simulation results and analysis of short-tube confinement

S3. Discussion about boundaries between positive and negative extra bending

S4. Simulation results of bending energies in polymer knots without excluded-volume (EV) interactions 


\section{S1. Additional simulation results and analysis of long-tube confinement}

Figure S1 shows the extra bending energies per persistence length in straight long-tube confinement. Here, the extra bending energy is defined as the difference in the bending energy between a confined polymer (DNA) and a polymer (DNA) in free space. The curves for different persistence lengths collapse. Such collapse is not surprising. In strong confinement, i.e. $D_{\text {tube }} / L_{p}<1$, the polymer conformations are substantially stretched, and the excluded volume interactions becomes negligible. The physical quantities of the polymer are dominated by the competition of the bending energy $\left(L_{p}\right)$ and the tube confinement $\left(D_{\text {tube }}\right)$.

Figure $\mathbf{S 2}$ shows the differences in the extra bending energy per bead between a curved long tube and a straight long tube with the same tube diameter. To understand the results, we consider a simple case: $D_{\text {tube }}=0$. For a straight long tube with $D_{\text {tube }}=0$, the bending energy is zero. For a curved long tube with $D_{\text {tube }}=0$, the polymer assumes a conformation following the shape of the tube axis (circle) and the bending energy per bead is $\frac{1}{2} \frac{L_{p}}{a}\left(\frac{a}{R_{c}}\right)^{2}$. Then, the difference is $\frac{1}{2} \frac{L_{p}}{a}\left(\frac{a}{R_{c}}\right)^{2}$ for $D_{\text {tube }}=0$. As we gradually increase $D_{\text {tube }}$ from zero, the difference becomes smaller than $\frac{1}{2} \frac{L_{p}}{a}\left(\frac{a}{R_{c}}\right)^{2}$. As the tube diameter becomes comparable with the persistence length, the difference in the bending energy becomes significantly less than $\frac{1}{2} \frac{L_{p}}{a}\left(\frac{a}{R_{c}}\right)^{2}$. These results suggest that we cannot treat polymer bending in curved tubes as the sum of polymer bending in straight tubes and the bending of tube axis.

Figure $\mathbf{3} 3$ shows the simulation results of the confinement free energy as a function of the tube diameter in straight long tubes, which agree with the Odijk scaling. The confinement free energy determines the entropic pulling force for polymers in short tubes.
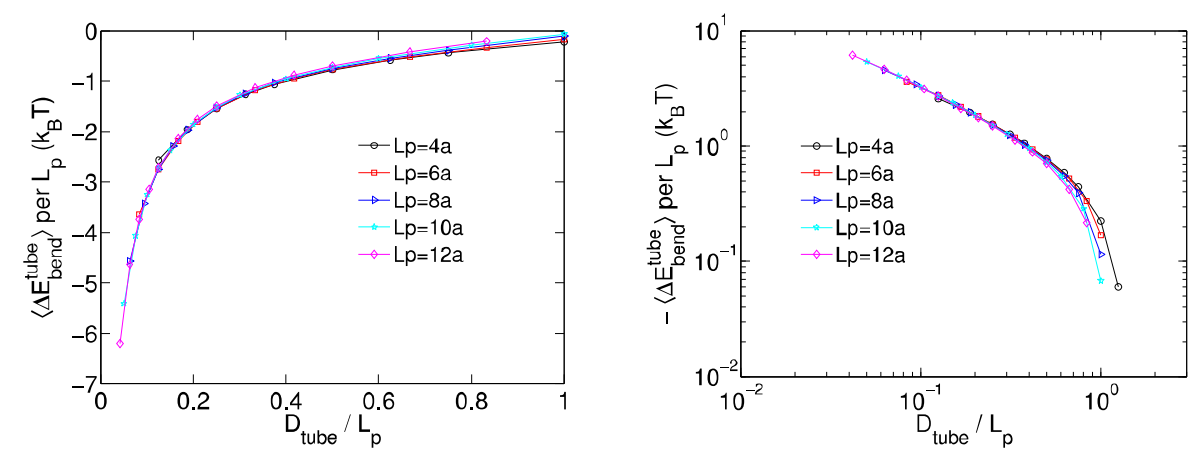

Figure S1. The extra bending energy per persistence length in long-tube confinement. (Left) In a linear-linear plot. (Right) In a log-log plot. 


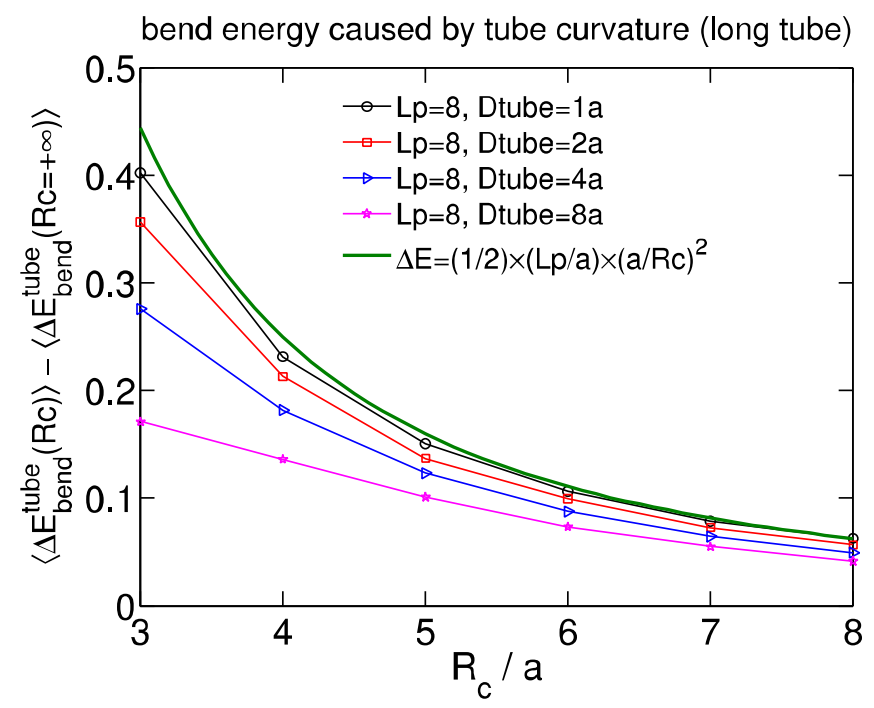

Figure S2. Simulation results of the differences in the extra bending energy per bead between a curved long tube and a straight long tube with the same tube diameter. The green line corresponds to the case of a perfect circle.

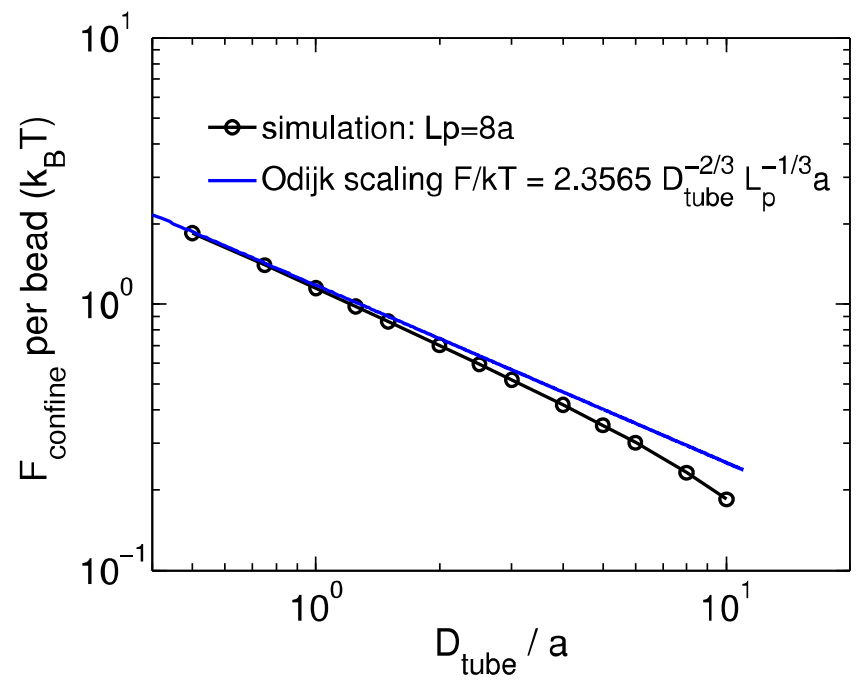

Figure S3. The confinement free energy per bead as a function of the tube diameter in long-tube confinement. The simulation results agree with the Odijk scaling. 


\section{S2. Additional simulation results and analysis of short-tube confinement}

Figure S4 illustrate a portion of a ring polymer (DNA) confined in a straight short tube. The short-tube confinement reduces the bending energies of polymer segments inside the tube, as described in the main text of the manuscript.

Figure $\mathbf{S} \mathbf{5}$ compares the bending energies and extensions from simulations using different tube lengths and polymer lengths. The results suggest that the bending energies and extensions are insensitive the tube length and polymer length when the tube only confines a portion of the polymer. The results are reasonable because the entropic pulling forces come from tube ends and do not depend on the tube length and polymer length.

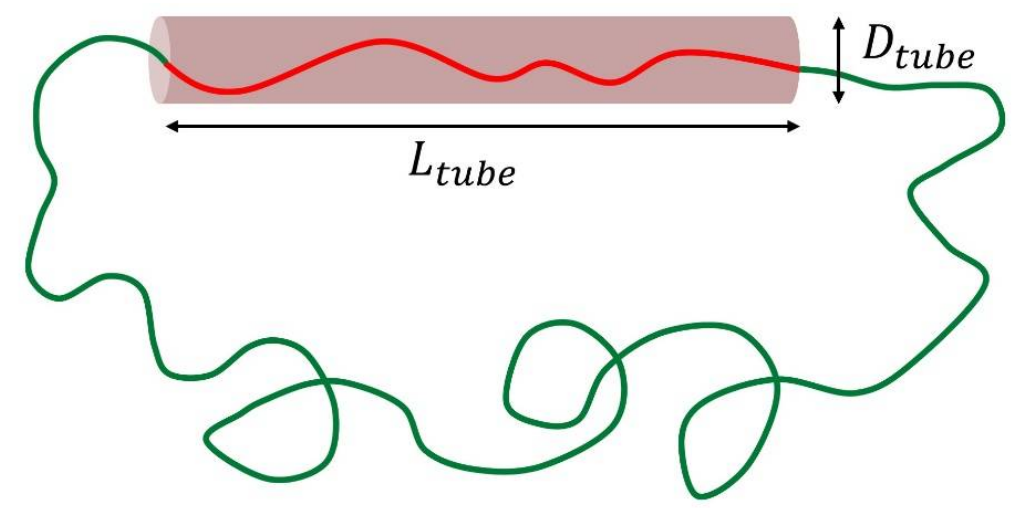

Figure S4. Illustration of a portion of a ring polymer (DNA) confined in a straight short tube.
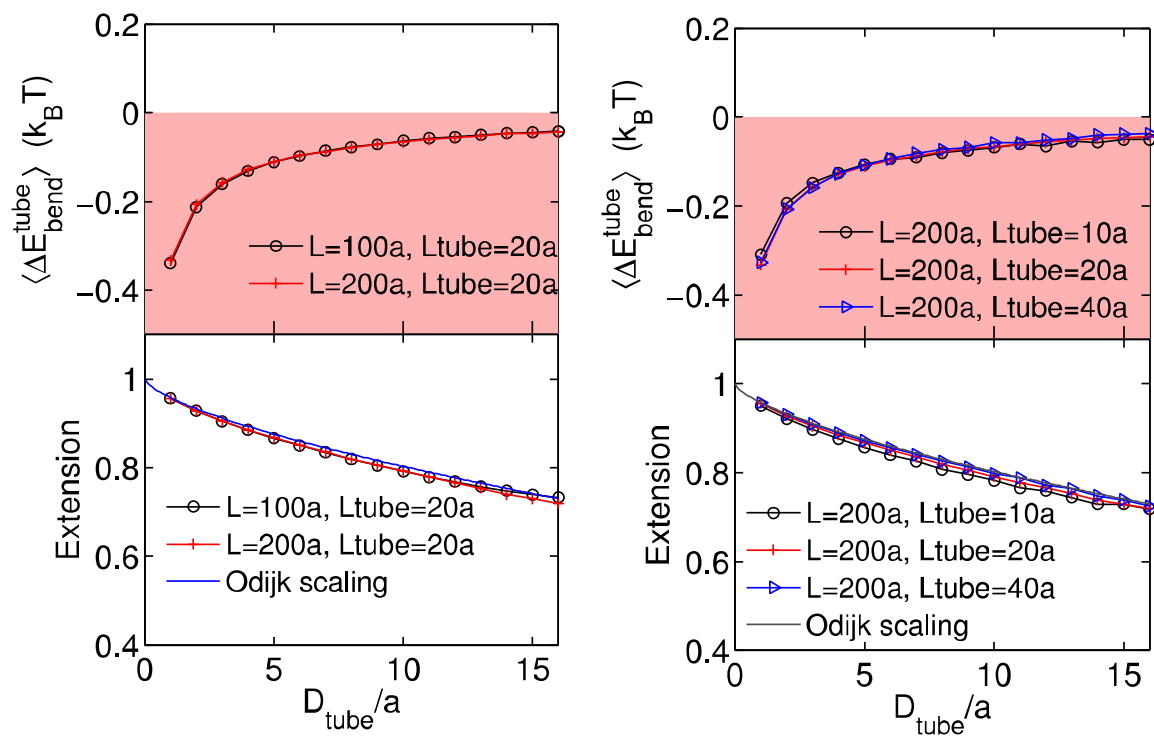

Figure 55. Simulation results of the extra bending energy and relative extensions in short tubes. (Left) Two lines correspond to different polymer lengths. The tube length is fixed as 20a. (Right) Three lines correspond to different tube lengths. The polymer length is fixed as 200 . 


\section{S3. Discussion about boundaries between positive and negative extra bending}

Figure $\mathbf{S 6}$ shows the critical tube diameter as a function of the radius of curvature of tube from short-tube simulations. Recall that the critical tube diameter $D_{\text {tube }}^{*}$ corresponds to zero extra bending energy. Three curves for three $L_{p}$ collapse after normalization of $D_{t u b e}^{*}$ and $R_{c}$ by $L_{p}$. We fit the three curves using the polynomial expression:

$$
y=-2.87302 x^{3}+6.49603 x^{2}-2.16254 x+0.17952
$$

In the above equation, y corresponds to $D_{\text {tube }}^{*} / L_{p}$, and $x$ corresponds to $R_{c} / L_{p}$.

We define a special value for the radius of curvature of tube, $R_{c 0}$, which corresponds to $D_{\text {tube }}^{*}=0$ (see illustration in Figure S6). We estimated $R_{c 0}$ using the following approach. In the case of $R_{c}=R_{c 0}$ and $D_{\text {tube }}=0$, the polymer conformation assumes an exact circular shape with a radius of $R_{c 0}$, and the bending energy per bead (per unit length $a$ ) is

$$
\left\langle E_{\text {bend }}^{\text {circle }}\right\rangle / k_{B} T=\frac{1}{2} \frac{L_{p}}{a}\left(\frac{a}{R_{c 0}}\right)^{2}
$$

Recall that $D_{\text {tube }}^{*}$ is definite based on $\left\langle\Delta E_{\text {bend }}\right\rangle=0$. Hence, one has

$$
\left\langle E_{\text {bend }}^{\text {circle }}\right\rangle=\left\langle E_{\text {bend }}^{\text {free }}\right\rangle
$$

where $\left\langle E_{\text {bend }}^{\text {free }}\right\rangle$ is the average bending energy per bead in free space. Combining Eq (S2) and Eq (S3), one has

$$
\frac{R_{c 0}}{L_{p}}=\sqrt{\frac{1}{2} \frac{a}{L_{p}\left\langle E_{\text {bend }}^{\text {free }}\right\rangle}}
$$

The value of $\left\langle E_{\text {bend }}^{\text {free }}\right\rangle$ can be approximated through the Boltzmann averaging of all bending angles (Figure S7):

$$
\left\langle E_{\text {bend }}^{\text {free }}\right\rangle / k_{B} T=\frac{\int_{0}^{\pi} E_{b} \sin \theta \exp \left(-E_{b} / k_{B} T\right) \mathrm{d} \theta}{\int_{0}^{\pi} \sin \theta \exp \left(-E_{b} / k_{B} T\right) \mathrm{d} \theta} \text { with } E_{b} / k_{B} T=\frac{1}{2} \frac{L_{p}}{a} \theta^{2}
$$

The above equation is rigorous for an isolated bending angle, i.e. three beads (Figure S7). In a polymer chain, the non-local interactions slightly affects $\left\langle E_{\text {bend }}^{\text {free }}\right\rangle$. Figure $\mathbf{5 8}$ shows the values of $\left\langle E_{\text {bend }}^{\text {free }}\right\rangle$ calculated from Eq. (S5) and from simulations, which agree with each other.

Substituting the values of $\left\langle E_{\text {bend }}^{\text {free }}\right\rangle$ into Eq (S4), we obtained the values of $R_{c 0}$ as a function of $L_{p}$ (Figure S8). The values of $R_{c 0}$ estimated from Eq (S4) agree with the data in Figure S6. 


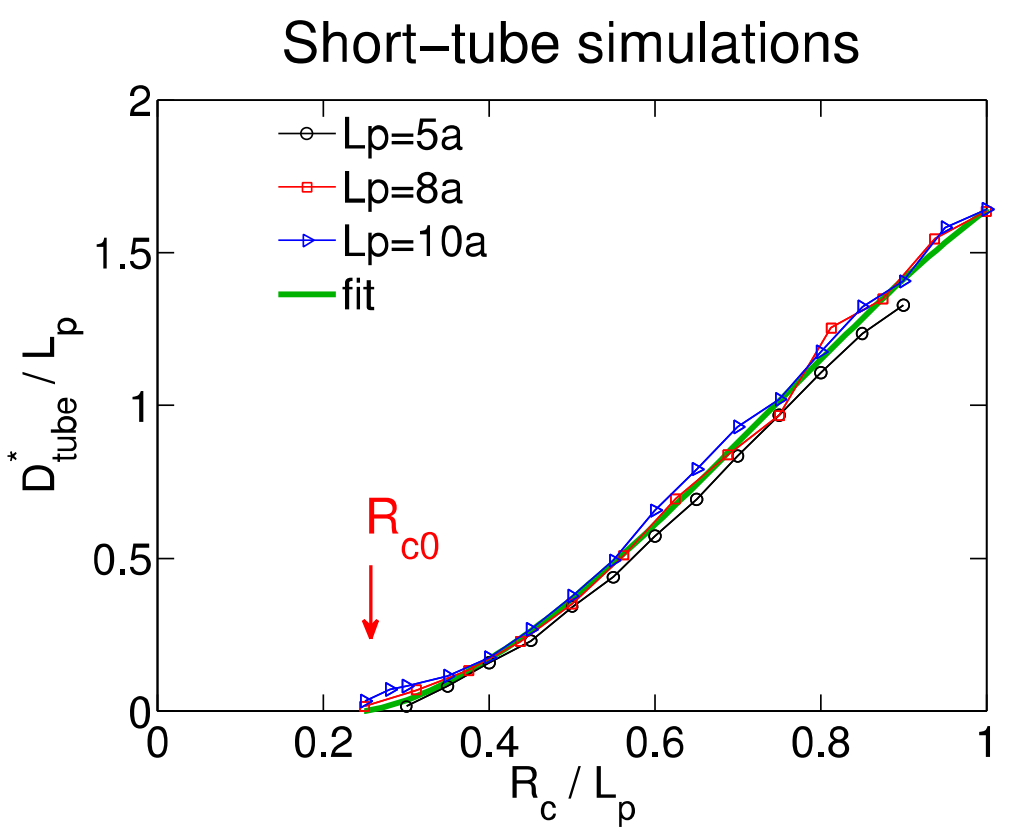

Figure S6. The critical tube diameter as a function of the radius of curvature of the tube from short-tube simulations. We define $R_{c 0}$ as the radius of curvature of tube corresponding to $D_{\text {tube }}^{*}=0$.

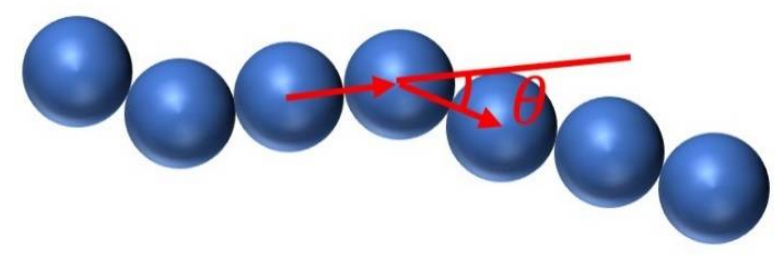

Figure S7. Illustration of the bending angle for three adjacent beads.
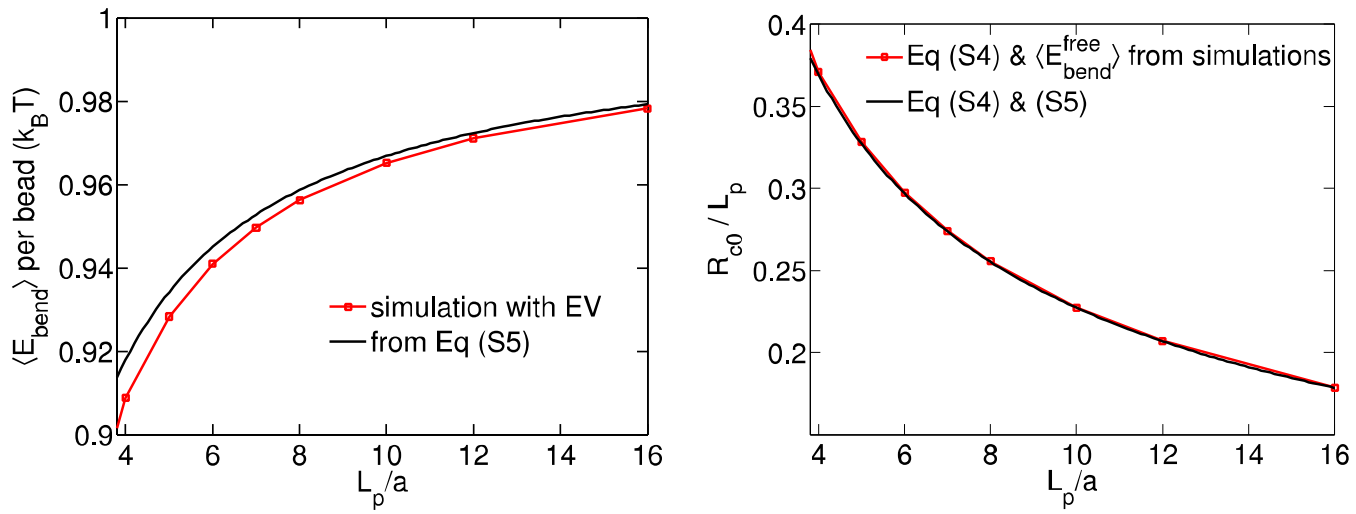

Figure S8. (Left) The average bending energy per bead in free space. Red symbols are from the simulation with EV interactions. The solid line is calculated from Eq (S5). (Right) The value of $R_{c 0}$ as a function of $L_{p}$. 
S4. Simulation results of bending energies in polymer knots without excluded-volume (EV) interactions

Figure $\mathbf{S 9}$ shows the extra bending energy of a trefoil knot as a function of the knot size in the simulations without excluded volume interaction. Three curves for three $L_{p}$ collapse after normalizing $L_{k n o t}$ by $L_{p}$. If we treat the semiflexible chains in these simulations as discretized worm-like chains, and the extra bending energies of knots in discretized worm-like chains are insensitive to the discretization when $L_{p} \geq 5 a$.

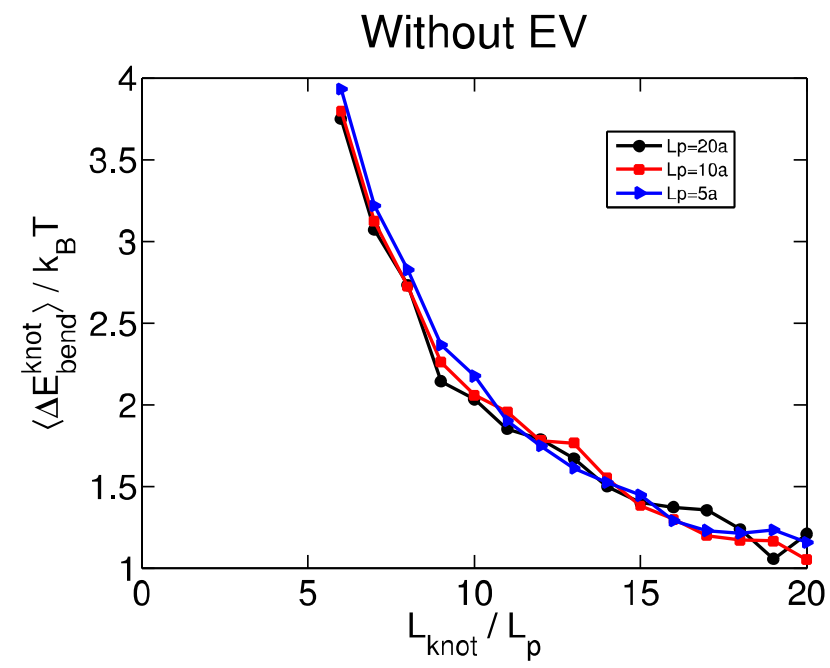

Figure S9. The extra bending energy of a trefoil knot as a function of the knot size. Three curves correspond to simulation results using a fixed polymer length $L=$ $1000 a$ and different persistence lengths. Excluded-volume interactions are turned off in these simulations. 